\title{
ISO-SWS spectra of Planetary Nebulae with low-mass [WC] central stars: a mixed C- and O-rich dust chemistry
}

\author{
Patrick W. Morris ${ }^{1,2}$, L.B.F.M. Waters ${ }^{1}$, and Douwe A. Beintema ${ }^{3}$ \\ ${ }^{1}$ Astronomical Institute, University of Amsterdam, \\ Kruislaan 403, NL-1098 SJ Amsterdam, the Netherlands \\ ${ }^{2}$ SRON, Sorbonnelaan 2, NL-3584CA Utrecht, the Netherlands \\ ${ }^{3}$ SRON, Landleven 12, NL-9700 AV Groningen, the Netherlands
}

\section{The mixed chemistry of [WC] dust shells}

The timing of $\mathrm{PNe}$ formation around low-mass $\mathrm{WC}$ stars is unsettled with respect to pulsations early in the post-AGB phase, or later thermal pulses (e.g., Tylenda $\&$ Gorny 1997). The chemistry of the dust in the nebulae can be used to trace the mass-loss history. Using ISO-sws spectroscopy, the PNe BD $+30^{\circ} 3639$ [WC9] and $\mathrm{He} 2-113$ [WC11] have been identified by Waters et al. (1998) to exhibit emission from $C$-rich dust (PAHs) in the surrounding envelopes at $\lambda<15 \mu \mathrm{m}$, while $O$-abundant silicate features are present at longer wavelengths. Figure 1 shows the PAH features, which include additional WCPNe observations to extend the range of stellar spectral subtypes.

Crystalline silicate features are also present beyond $20 \mu \mathrm{m}$, suggesting that the O-rich phase of the central stars underwent strong mass-loss at or near the end of the AGB phase. This was probably followed by a change to C-rich chemistry at the surface as traced by the PAHs.

\section{Basic implications}

A dust model for $\mathrm{BD}+30^{\circ} 3639$ (Waters et al. 1998) indicates that the mass of the C-rich shell is about the same as the $\mathrm{H}+\mathrm{He}$ layers of many post-AGB stars, suggesting that the post-AGB pulse is responsible for exposing the He- and Crich inner layers. The persistence of PAHs in all observed envelopes indicates that pulsed ejection of the C-rich layers is most likely to have occured no later than the [WCL] phase, when surface H may still be present (e.g., Hamann 1997). Because of the H-free chemistry at [WCE] surfaces (NGC 6369 in our sample), formation of the PAH-bearing shell during later thermal pulsing is unlikely. The dynamical age $(\sim 1050 \mathrm{yr})$ of the $\mathrm{O}$-rich shell around $\mathrm{BD}+30^{\circ} 3639$ suggests that the chemistry change was also initated by a post-AGB pulse rather than by a late thermal pulse (Waters et al. 1998). 


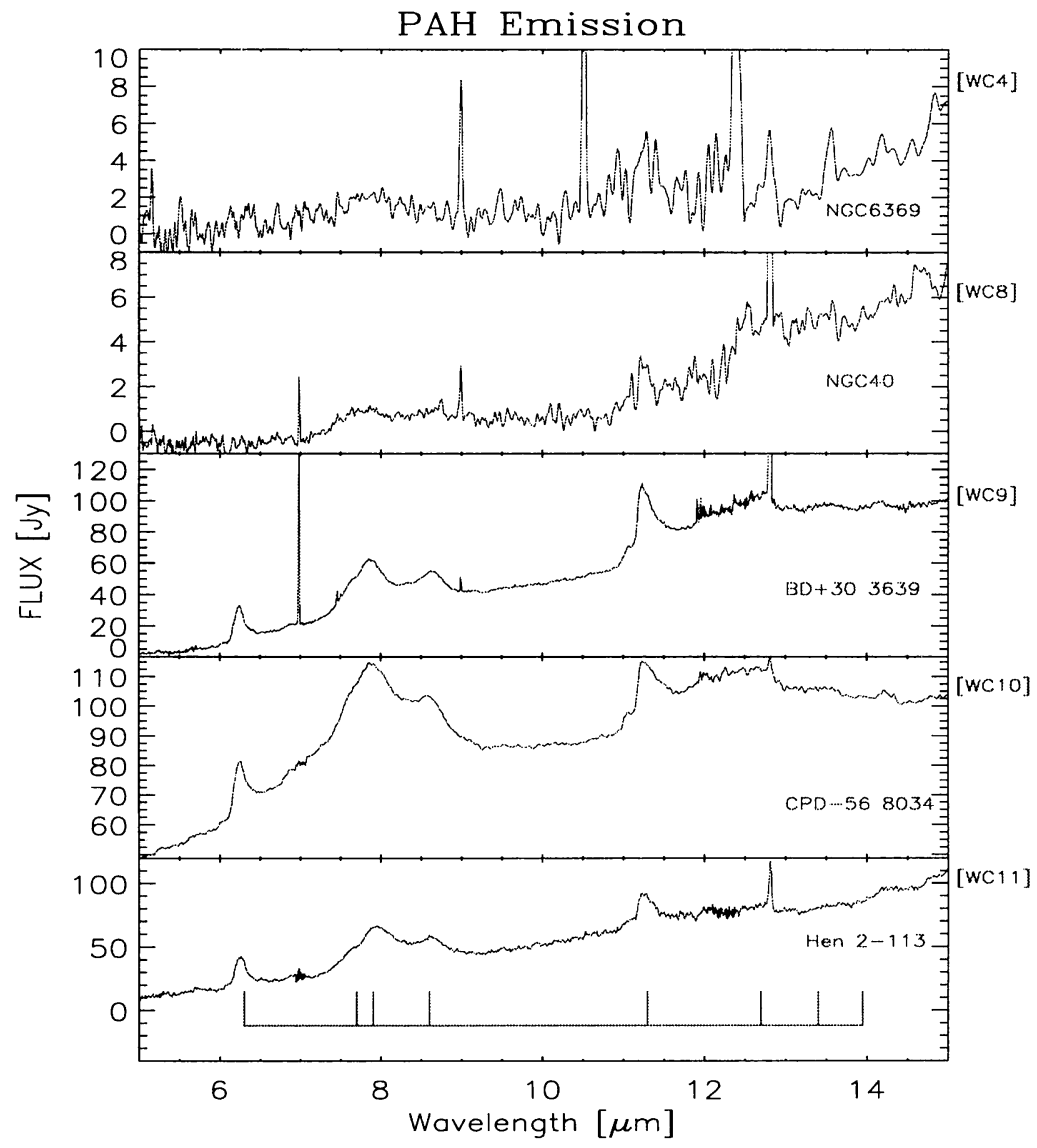

Figure 1. Persistent presence of the family of UIR bands in ISO-SWS [WC] spectra, associated with $\mathrm{H}$ - and $\mathrm{C}$-rich $\mathrm{PAH}$ molecules.

\section{References}

Hamann, W.-R. 1997, in: H.J. Habing \& H. Lamers (eds.), Planetary Nebulae, Proc. IAU Symp. No. 180 (Kluwer: Dordrecht), p. 91

Tylenda, R., Górny, S.K. 1997, in: H.J. Habing \& H. Lamers (eds.), Planetary Nebulae, Proc. IAU Symp. No. 180 (Kluwer: Dordrecht), p. 371

Waters, L.B.F.M., Beintema, D.A., Zijlstra, A.A., de Koter, A., Molster, F.J., Bouwman, J., de Jong, T., Pottasch, S.R., de Graauw, Th. 1998, A\&A 331, L61 